\title{
Eficácia, segurança e aspectos regulatórios dos medicamentos órfãos para doenças raras: o caso Zolgensma ${ }^{\circledR}$
}

\author{
Efficacy, safety and regulatory aspects of orphan drugs for rare diseases: Zolgensma ${ }^{\circledR}$ 's case \\ study
}

Eficacia, seguridad y aspectos regulatorios de medicamentos huérfanos para enfermedades raras: o caso de Zolgensma ${ }^{\circledR}$

Rachel Riera ${ }^{1}$

Ângela Maria Bagattini²

Daniela Pachito 3

\begin{abstract}
Resumo
Objetivo: discutir o processo regulatório de medicamentos para doenças raras no Brasil, com base no caso Zolgensma ${ }^{\circledR}$, e avaliar criticamente as evidências disponíveis até 0 momento sobre a eficácia e a segurança do Zolgensma ${ }^{\circledR}$ no tratamento da atrofia muscular espinhal (AME). Metodologia: estudo descritivo realizado no Núcleo de Avaliação de Tecnologias em Saúde do Hospital Sírio-Libanês (NATS-HSL) em junho de 2019. Resultados: em abril de 2019, o uso do Zolgensma ${ }^{\circledR}$ para AME foi regulamentado nos Estados Unidos com base em dois estudos clínicos abertos (sem mascaramento), sem grupo comparador paralelo (e, portanto, não randomizados). Essas limitações metodológicas aumentam a incerteza nos resultados encontrados. A Agência Nacional de Vigilância Sanitária (Anvisa) aprovou estratégias para regulamentar o processo e os prazos de análise das submissões de registro de novos medicamentos para doenças raras, caso do Zolgensma ${ }^{\circledR}$. Conclusão: faz-se necessário ampliar o debate em torno do processo de regulamentação e de incorporação de medicamentos órfãos para doenças raras no Brasil. $O$ debate deve incluir as evidências relacionadas aos efeitos - benefícios e riscos - desses medicamentos, e maior clareza nos critérios para concessão de registro e recomendação de incorporação em sistemas de saúde.
\end{abstract}

Palavras-chaves: Judicialização da saúde. Atrofia muscular espinal. Doenças raras. Terapia genética. Doenças neuromusculares.

\footnotetext{
Abstract

Objective: to discuss the regulatory process of drugs for rare diseases in Brazil, based on the Zolgensma ${ }^{\circledR}$ case, and to critically evaluate the evidence so far available on the efficacy and safety of Zolgensma ${ }^{\circledR}$ for treating spinal muscular atrophy (SMA). Methods: descriptive

${ }^{1}$ Coordenadora do Núcleo de Avaliação de Tecnologias em Saúde do Hospital Sírio-Libanês; professora na Escola Paulista de Medicina pela Universidade Federal de São Paulo, São Paulo, SP. E-mail: rachel.riera@hsl.org.br

2 Pesquisadora do Núcleo de Avaliação de Tecnologias em Saúde do Hospital Sírio-Libanês, São Paulo, SP. E-mail: angela.mbagattini@hsl.org.br

${ }^{3}$ Pesquisadora do Núcleo de Avaliação de Tecnologias em Saúde do Hospital Sírio-Libanês, São Paulo, SP. E-mail: daniela.vpachito@hsl.org.br
} 
study conducted at the Health Technology Assessment Center of the Sírio Libanês Hospital (NATS-HSL) in June 2019. Results: in April 2019, the use of Zolgensma ${ }^{\circledR}$ for SMA was regulated in the United States based on two open-lable (unmasked), non-comparative (and therefore non-randomized) studies. These methodological limitations increase the uncertainty related to study results. The National Agency of Sanitary Surveillance (Anvisa) has approved strategies to regulate the process and specific deadlines for completing the appraisal process of new medicines for rare diseases, such as Zolgensma ${ }^{\circledR}$. Conclusion: it is necessary to broaden the debate about the process of regulation and incorporation of orphan drugs for rare diseases in Brazil. This debate should include evidence related to the effects (benefits and risks) of these drugs, and greater transparency of the criteria indispensable for granting registration and recommendation of incorporation into health systems.

Keywords: Health's judicialization. Muscular atrophy spinal. Rare diseases. Gene therapy. Neuromuscular diseases.

\section{Resumen}

Objetivo: analizar el proceso de fármacos reguladores para enfermedades raras en Brasil, con base en el caso Zolgensma, y para evaluar críticamente la evidencia disponible hasta el momento sobre la eficacia y seguridad de Zolgensma ${ }^{\circledR}$ nel tratamiento de atrofia muscular espinal (AME). Métodos: estudio descriptivo realizado en el Centro de Evaluación de Tecnología de Salud del Hospital Sírio-Libanês (NATS-HSL) en junio de 2019. Resultados: en abril de 2019, el uso de Zolgensma ${ }^{\circledR}$ para la AME se reguló en los Estados Unidos basado en dos estudios abiertos (no enmascarados), no comparativos (y por lo tanto no aleatorios). Estas limitaciones metodológicas aumentan la incertidumbre en los resultados encontrados. La Agencia Nacional de Vigilancia Sanitaria en Brasil (Anvisa) ha aprobado estrategias para regular el proceso y los plazos de análisis de las solicitudes de registro de nuevos fármacos para enfermedades raras, el caso de Zolgensma. Conclusión: es necesario ampliar el debate sobre el proceso de regulación e incorporación de medicamentos huérfanos para enfermedades raras en Brasil. Este debate debe incluir evidencia relacionada con los efectos (beneficios y riesgos) de estos medicamentos y una mayor transparencia en los criterios para otorgar el registro y la recomendación de incorporación a los sistemas de salud.

Palabras-llave: Judicialización de la salud. Atrofia muscular espinal. Enfermedades raras. Terapia genética. Enfermedades neuromusculares

\section{Introdução}

Um novo medicamento para o tratamento de crianças com atrofia muscular espinhal (AME) acaba de receber aprovação da U.S Food and Drug Administration (FDA) (1). Zolgensma $^{\circledR}$ (onasemnogene abeparvovec-xioi) é a primeira terapia gênica, que consiste na substituição de genes mutados por genes sadios, aprovada para a AME, sendo aplicada em uma única dose por paciente, cujo custo ultrapassa o valor de oito milhões de reais.

A AME é uma doença genética autossômica recessiva que acomete um em cada 10 a 11 mil recém-nascidos e está associada à elevada mortalidade nos dois primeiros anos de 
vida $(2,3)$. No Brasil, estima-se uma incidência de 250 a 300 novos casos por ano, com um total de 3.800 casos diagnosticados atualmente. O CID10-G12, atrofia muscular espinal e síndromes correlatas, tem 113 óbitos registrados no Sistema de Informações sobre Mortalidade (SIM) entre 2010 e 2017, com uma média de 14 óbitos/ano por essa causa (4).

Há cinco subtipos de AME, que se diferem principalmente quanto à idade de surgimento e à gravidade do quadro clínico $(5,6)$. De modo geral, todos eles se caracterizam por fraqueza muscular, atrofia e, nos casos mais graves, por paralisia, insuficiência respiratória e morte prematura (antes dos dois anos de idade), observada em aproximadamente $68 \%$ dos casos $(7,8)$.

O tratamento da AME inclui principalmente medidas de suporte, como fisioterapia motora e respiratória, e cuidados nutricionais. O primeiro medicamento comercializado especificamente para AME, o Spinraza ${ }^{\circledR}$ - nusinersena, uso intratecal -, já foi regulamentado no Brasil e será incorporado na Relação Nacional de Medicamentos Essenciais (Rename) do Sistema Único de Saúde (SUS). Com avaliação da Conitec, o medicamento foi incorporado no SUS para AME do tipo $1^{4}$, e para os pacientes com AME tipos 2 e tipo 3, o acesso é mediante acordo de compartilhamento de risco (projeto piloto). ${ }^{5}$

Considerando que o Zolgensma ${ }^{\circledR}$ é uma tecnologia com possibilidade de registro no Brasil, e pode ser a primeira terapia gênica a entrar no mercado nacional, este texto tem como objetivo discutir o processo regulatório de medicamentos para doenças raras no Brasil, com base no caso Zolgensma ${ }^{\circledR}$, e avaliar criticamente as evidências disponíveis até o momento sobre a eficácia e a segurança do Zolgensma ${ }^{\circledR}$ no tratamento da AME.

\section{Métodos}

Estudo descritivo realizado no Núcleo de Avaliação de Tecnologias em Saúde do Hospital Sírio-Libanês (NATS-HSL) em junho de 2019.

\footnotetext{
${ }^{4}$ Portaria no 24/SCTIE/MS, de 24 de abril de 2019), consoante recomendação da Comissão Nacional de Incorporação de Tecnologias no SUS (Conitec).

${ }^{5}$ Portaria GM n 1.297, de 11 de junho de 2019.
} 


\section{Resultados e Discussão}

\section{Aspectos regulatórios do Zolgensma ${ }^{\circledR}$ na FDA}

Em 1ํ de outubro de 2018, a AveXis Inc submeteu oficialmente o pedido de licença de biológicos (registro BLA 125694) do Zolgensma ${ }^{\circledR}$ para análise da FDA, especificando seu uso para o tratamento de população pediátrica, com menos de dois anos de idade, com diagnóstico de atrofia muscular espinhal e mutação bialélica no gene do neurônio motor de sobrevivência 1 (SMN1). No entanto, todo o processo regulatório do Zolgensma ${ }^{\circledR}$ foi iniciado sete anos antes da concessão do registro, em dezembro de 2011. Sequencialmente, o processo regulatório incluiu: autorização para seguir sob aprovação rápida (fast track), em 2013; classificação como medicamento órfão, em 2014; designação como terapia avançada, em 2016; indicação para doença pediátrica rara, em 2018; primeira submissão, em outubro de 2018; classificação do processo como prioritário pelo comitê de revisão da FDA, em novembro de 2018; entrega de dados atualizados de eficácia e segurança, em fevereiro 2019; e entrega de dados adicionais de eficácia e segurança do estudo clínico fase $3 \mathrm{em}$ andamento, em abril de 2019). Nas etapas futuras, a AveXis conduzirá estudos para coletar dados de eficácia e segurança dos participantes dos dois estudos clínicos, fases 1 e 3 , durante um período de seguimento de 15 anos após o tratamento, por meio de visitas anuais nos primeiros cinco anos e depois por contato telefônico anual por 10 anos. Outras medidas de farmacovigilância também foram propostas pelo fabricante. ${ }^{6}$

De acordo com divulgação da FDA, aproximadamente um mês após a aprovação do medicamento, a agência foi informada pela AveXis Inc. sobre um problema de manipulação de dados dos testes do produto realizados em animais na fase pré-clínica dos estudos ${ }^{7}$. Essa informação não fez com que a FDA retirasse a aprovação do medicamento, pois consideraram que isso não alterou os resultados positivos que sustentam o perfil de risco/benefício dos estudos em humanos. Contudo, a FDA tomará medidas apropriadas, pois o fabricante sabia dessa informação antes da aprovação do medicamento. Além disso, para proteção dos pacientes, profissionais da saúde e outros envolvidos, os dados estão sendo

\footnotetext{
${ }^{6}$ O processo detalhado pode ser acessado em https://www.fda.gov/media/127961/download.

7 Declaração da FDA: Statement on data accuracy issues with recently approved gene therapy em: https://www.fda.gov/news-events/press-announcements/statement-data-accuracy-issues-recently-approved-genetherapy?utm_campaign=080619_Statement_Statement\%20by\%20FDA\%20on\%20data\%20accuracy\%20issues\%20with \%20gene\%20therapy\&utm_medium=email\&utm_source=Eloqua
} 
avaliados para garantir dados verdadeiros, completos e precisos.

Aspectos regulatórios para medicamentos para doenças raras no Brasil

A Agência Nacional de Vigilância Sanitária (Anvisa), por meio da Resolução da Diretoria Colegiada (RDC) 205/2017, aprovou estratégias para reduzir os prazos de análise das submissões de registro de novos medicamentos para doenças raras (9). Até então, não havia regulamentação específica para aprovação de ensaios clínicos; confirmação da adesão às boas práticas em pesquisa; e fabricação e registro de novas tecnologias para doenças classificadas como raras. Alguns critérios, exigidos fora do contexto de doenças raras, foram modificados, mas buscando manter a qualidade na avaliação da eficácia e da segurança das tecnologias. A apresentação de dados adicionais posteriormente à concessão do registro passou a ser permitida por meio de assinatura de termo de compromisso entre a Anvisa e a empresa solicitante. Para medicamentos importados, a necessidade de testes de controle de qualidade no Brasil foi descartada, desde que o teste existente tenha sido realizado pelo fabricante e que seja comprovada a manutenção de condições apropriadas durante o transporte do medicamento (9).

Para situações nas quais não há alternativa terapêutica disponível ou quando há benefício significativo com o uso da intervenção, a Anvisa segue uma estratégia de priorização para avaliar solicitações de alteração pós-registro (como ampliação de uso ou nova indicação terapêutica) de medicamentos para doenças raras dentro de um prazo de 120 dias (RDC no 204/2017) (10).

No Brasil, medicamentos com eficácia comprovada no tratamento ou diagnóstico de doenças raras, com incidência menor que 5:10.000, cuja dispensação atende a casos específicos, são denominados medicamentos órfãos (11). Esse é o caso do Zolgensma ${ }^{\circledR}$.

\section{Aspectos de eficácia e segurança}

Como já apresentado, o Zolgensma ${ }^{\circledR}$, administrado em dose única intravenosa, está indicado para crianças com AME, com até dois anos de idade, e com mutação bialélica no gene SMN1 (12). Os efeitos do medicamento para esse subgrupo foram avaliados por um 
estudo clínico de fase 1 já concluído ${ }^{8}$ e por um estudo clínico de fase 3 em andamento ${ }^{9}$, que tiveram seus resultados comparados com controles históricos, originados de estudos de coorte sobre a história natural da doença, incluindo participantes que receberam exclusivamente a terapia de suporte. Esses dois estudos formam a base para o processo de submissão e aprovação na FDA.

No estudo clínico de fase 1,15 crianças com AME, com média de idade de 4 meses, foram divididas para receber uma única dose alta $(n=12)$ ou baixa $(n=3)$ de Zolgensma ${ }^{\circledR}$. Aos 20 meses de idade, todas elas estavam vivas e sem uso de ventilação mecânica permanente; no coorte histórico que serviu de grupo controle, essa taxa foi de $8 \%$. No grupo que recebeu dose alta, houve melhora da função motora ao longo do estudo; no coorte histórico, houve piora da função motora ao longo do tempo. Ao final do estudo, alguns marcos motores foram alcançados pelas crianças que receberam Zolgensma ${ }^{\circledR}$, como sentar sem ajuda, manter alimentação via oral, rolar e caminhar de modo independente. Esses marcos não foram atingidos na mesma proporção pelas crianças do coorte histórico (13) (14).

No estudo fase 3 em andamento, 21 crianças receberam dose alta de Zolgensma ${ }^{\circledR}$. De acordo com a análise feita em março de 2019, 19 pacientes estavam vivos sem ventilação mecânica permanente, um paciente morreu aos 7,8 meses de idade devido à progressão da doença, e houve uma perda: um participante saiu do estudo aos 11,9 meses de idade. Nessa análise, $47,6 \%$ dos participantes conseguia sentar-se sem apoio por $\geq 30$ segundos, entre 9,2 e 16,9 meses de idade. Com base na história natural da doença, não era esperado que as crianças que entraram no estudo conseguissem permanecer sentadas sem apoio; e era esperado que apenas $25 \%$ delas estivessem vivas e sem uso de ventilação mecânica após os 14 meses de idade (15).

Além desses dois estudos, que foram utilizados como documentação para solicitar o registro nas agências regulatórias internacionais, foram identificados outros seis estudos em andamento sobre os benefícios e riscos do Zolgensma ${ }^{\circledR}$ na base clinicaltrials.gov ${ }^{10}$ :

i. NCT03461289, com 30 crianças, ainda não recrutando (16);

\footnotetext{
${ }^{8}$ Estudo CL-101, NCT02122952

${ }^{9}$ Estudo CL-303, NCT03306277

10 https://clinicaltrials.gov/
} 
ii. NCT03421977, extensão do estudo CL-101com os mesmos participantes (17);

iii. NCT03505099, com 27 crianças de até 6 semanas de vida, recrutando (18);

iv. NCT03837184, com 6 crianças de até seis meses de vida, recrutando (19);

v. NCT03955679, coorte aberta, participantes de qualquer idade (20);

vi. NCT03381729, com 27 crianças, entre 6 e 60 meses de vida, recrutando, uso de Zolgensma $^{\circledR}$ intratecal (21).

A discussão em torno da aprovação do Zolgensma ${ }^{\circledR}$ pela FDA levanta uma discussão paralela sobre os critérios metodológicos assumidos por agências regulatórias mundiais para aprovação da comercialização de medicamentos, especificamente no cenário de doença raras, e medicamentos órfãos, com custos extremamente elevados. Nesse sentido, podem ser identificados pontos críticos relacionados aos métodos usados na condução dos estudos clínicos que embasaram o processo de regulamentação do Zolgensma ${ }^{\circledR}$ na FDA. Tais pontos podem ter repercussões na qualidade dos resultados desses estudos e na certeza relacionadas ao corpo final das evidências existentes até o momento.

Primeiramente, é possível observar que todos os estudos listados acima estão distantes do rigor metodológico de um ensaio clínico randomizado duplo-cego, considerado o desenho de estudo primário mais apropriado para avaliar efeitos de intervenções em saúde. São estudos abertos, sem mascaramento, sem grupo comparador paralelo e não são randomizados. O fato de estarmos lidando com uma doença rara é apontado, algumas vezes, como justificativa para a escolha de diferentes desenhos de estudo. A seguir se discutirá, do ponto de vista metodológico, os riscos aos quais se está vulnerável quando se aceita tal justificativa.

A presença de um grupo comparador, similar ao grupo que recebeu a intervenção, é fundamental para estimar o real efeito dessa intervenção e avaliar, por exemplo, se esses efeitos são diferentes daqueles observados com o uso de uma intervenção já disponível, com o uso do placebo ou com a evolução natural da doença. A similaridade entre os dois grupos ajuda a garantir que o efeito observado ao final do estudo seja devido ao uso ou não da intervenção, eliminando qualquer outro fator que diferencie os grupos, como gravidade da doença, sexo, idade ou presença de comorbidades.

Listados entre os mais de 50 tipos catalogados de riscos de viés, a randomização, o sigilo de alocação e o mascaramento são características que, quando ausentes, aumentam 
a probabilidade de que os resultados de um estudo sejam desviados da verdade (22).

A randomização - distribuição aleatória dos participantes entre os grupos de estudos - permite que os grupos fiquem similares com relação a fatores conhecidos e não conhecidos, como mutações genéticas associadas a diferentes prognósticos da doença, e que possam influenciar os resultados.

O sigilo de alocação é definido como a manutenção da sequência de alocação (randomização) sigilosa até que a intervenção seja administrada. Na maioria das vezes isso é feito utilizando estratégias como central telefônica, sistema online, ou envelopes numerados, lacrados e opacos. A ausência de sigilo de alocação pode superestimar o tamanho do efeito da intervenção em $37 \%$ a $41 \%(23,24)$. Na prática, isso quer dizer que, dependendo da estimativa pontual do efeito, a direção desse efeito pode ser invertida, ou seja, uma intervenção que, na realidade, não apresenta benefícios, pode se mostrar falsamente benéfica em estudos que não tenham utilizado métodos apropriados para preservar o sigilo de alocação.

Estudos abertos - que não usam métodos de mascaramento de participantes, equipe dos estudos e avaliadores dos desfechos - estão susceptíveis a viés de performance e de aferição. Participantes e profissionais responsáveis pela administração da intervenção podem levar a desvios no processo de condução do estudo, como adesão do tratamento, relato de eventos adversos, etc, quando cientes da alocação; os avaliadores podem mensurar os desfechos de modo subjetivo, ao utilizarem ferramentas como questionários que avaliam qualidade de vida, dor e função física, por exemplo -, exames radiográficos, etc.

Uma avaliação mais detalhada desses estudos poderia identificar, pelos menos, alguns dos 51 tipos de vieses já identificados e catalogados (22).

Apesar dos potenciais benefícios observados nos estudos citados, há discussão sobre a relevância clínica dos desfechos escolhidos para a avaliação da eficácia, como a proporção de pacientes atingindo o marco de sentar-se sem apoio por pelo menos 30 segundos aos 18 meses de idade. Alguns deles foram, inclusive, redefinidos ao longo dos estudos, o que reduz a confiabilidade no processo de condução e análise dos dados. Também é importante lembrar que o Zolgensma ${ }^{\circledR}$ ainda não foi avaliado para casos avançados de AME, como paralisia completa dos membros, uso de ventilação mecânica de 
modo permanente, o que limita a extrapolação dos achados disponíveis até o momento para essa subpopulação. Seus efeitos de longo prazo e os efeitos associados ao uso de mais de uma dose ainda também não são conhecidos.

\section{Conclusões}

O Zolgensma ${ }^{\circledR}$ deve chegar em breve ao Brasil e outras terapias gênicas também são esperadas. Parece ser prioritário um debate entre a sociedade civil; profissionais da saúde e do direito; gestores; agências regulatórias e de incorporação; e associação de pacientes tanto sobre o processo regulatório de concessão de registro quanto sobre o processo de incorporação no sistema de saúde de medicamentos órfãos para doenças raras. Esse debate deveria idealmente incluir discussões sobre a incerteza relacionada aos benefícios e riscos conhecidos desses medicamentos; os critérios para a tomada de decisão em relação à concessão de registro e à recomendação de incorporação em sistemas de saúde; as estratégias de monitoramento de desempenho após a incorporação; e a possível judicialização da tecnologia. Estamos prontos para esse debate?

\section{Referências}

1. U.S Food \& Drug Administration. BL 125694/0, BLA Approval. 2019. Disponível em: https://www.fda.gov/media/126130/download [Acesso em 19 jun. de 2019]. (US-FDA 2019a)

2. Lunn MR, Wang $\mathrm{CH}$. Spinal muscular atrophy. Lancet (London, England) [Internet]. 2008 Jun 21;371(9630):2120-33. Disponível em:

https://linkinghub.elsevier.com/retrieve/pii/S0140673608609216 [Acesso em 10 jul. 2019].

3. Ogino S, Leonard DGB, Rennert H, Ewens WJ, Wilson RB. Genetic risk assessment in carrier testing for spinal muscular atrophy. Am J Med Genet [Internet]. 2002 Jul 15 110(4):3017. Disponível em: http://www.ncbi.nlm.nih.gov/pubmed/12116201 [Acesso em 10 jul. 2019].

4. Brasil. SIM-Sistema de Informações de Mortalidade[homepage on the Internet] [Internet]. DatasusDisponível em: http://datasus.saude.gov.br/sistemas-e-aplicativos/eventos-v/simsistema-de-informacoes-de-mortalidade [Acesso em 10 maio 2019]. 
5. Verhaart IEC, Robertson A, Wilson IJ, Aartsma-Rus A, Cameron S, Jones CC, et al. Prevalence, incidence and carrier frequency of $5 q$-linked spinal muscular atrophy - a literature review. Orphanet J Rare Dis [Internet]. 2017 Dec 4 12(1):124. Disponível em: http://ojrd.biomedcentral.com/articles/10.1186/s13023-017-0671-8 [Acesso em 10 jul. 2019].

6. Finkel R, Bertini E, Muntoni F, Mercuri E, ENMC SMA Workshop Study Group. 209th ENMC International Workshop: Outcome Measures and Clinical Trial Readiness in Spinal Muscular Atrophy 7-9 November 2014, Heemskerk, The Netherlands. Neuromuscul Disord [Internet]. 2015 Jul;25(7):593-602. Disponível em: http://www.ncbi.nlm.nih.gov/pubmed/26045156 [Acesso em 10 jul. 2019].

7. Zerres K, Rudnik-Schöneborn S. Natural history in proximal spinal muscular atrophy. Clinical analysis of 445 patients and suggestions for a modification of existing classifications. Arch Neurol [Internet]. 1995 May 52(5):518-23. Disponível em:

http://www.ncbi.nlm.nih.gov/pubmed/7733848 [Acesso em 10 jul. 2019].

8. Munsat T, Davies K. Spinal muscular atrophy. 32nd ENMC International Workshop. Naarden, The Netherlands, 10-12 March 1995. Neuromuscul Disord [Internet]. 1996 Mar 1 6(2):125-7. Disponível em: http://www.ncbi.nlm.nih.gov/pubmed/8664564 [ Acesso em 10 jul. 2019].

9. Ministério da Saúde - MS Agência Nacional de Vigilância Sanitária - Anvisa. RESOLUÇÃO DA DIRETORIA COLEGIADA - RDC № 205, DE 28 DE DEZEMBRO DE 2017. Disponível em: http://portal.anvisa.gov.br/documents/10181/2718376/RDC_205_2017_.pdf/996fc46e216b-44ab-b8c8-2778151b786e [Acesso em 02 jul. 2019]. (Ministério da Saúde 2017a)

10. Ministério da Saúde - MS Agência Nacional de Vigilância Sanitária - Anvisa. RESOLUÇÃO DA DIRETORIA COLEGIADA - RDC N²04, DE 27 DE DEZEMBRO DE 2017. Disponível em: http://portal.anvisa.gov.br/documents/10181/2718376/RDC_204_2017_.pdf/b2d4ae64-2d9144e9-ad67-b883c752c094 [Acesso em 02 jul. 2019]. (Ministério da Saúde 2017b)

11. Ministério da Saúde - MS Agência Nacional de Vigilância Sanitária - Anvisa. RESOLUÇÃO DA DIRETORIA COLEGIADA - RDC Nº, DE 28 DE AGOSTO DE 2006. Disponível em: http://www4.anvisa.gov.br/base/visadoc/CP/CP\%5B16120-1-0\%5D.PDF [Acesso em 02 jul. 2019]. (Ministério da Saúde 2017c)

12. U.S Food \& Drug Administration. Zolgensma. [Internet]. Disponível em: https://www.fda.gov/vaccines-blood-biologics/zolgensma [Acesso em 2019 jun. 19] (US-FDA 2019b).

13. Al-Zaidy S, Pickard AS, Kotha K, Alfano LN, Lowes L, Paul G, et al. Health outcomes in spinal muscular atrophy type 1 following AVXS-101 gene replacement therapy. Pediatr Pulmonol. 2019;54(2):179-85. doi: 10.1002/ppul.24203 
14. Mendell JR, Al-Zaidy S, Shell R, Arnold WD, Rodino-Klapac LR, Prior TW, et al. SingleDose Gene-Replacement Therapy for Spinal Muscular Atrophy. N Engl J Med. 2017;377(18):1713-22. Disponível em: http://www.nejm.org/doi/10.1056/NEJMoa1706198 [Acesso em 10 jul. 2019].

15. NCT03306277. Gene Replacement Therapy Clinical Trial for Patients With Spinal Muscular Atrophy Type 1 (STR1VE). Disponível em: https://clinicaltrials.gov/ct2/show/NCT03306277 [Acesso em 19 jul. 2019].

16. NCT03461289. ClinicalTrials.gov. Single-Dose Gene Replacement Therapy Clinical Trial for Patients With Spinal Muscular Atrophy Type 1 (STRIVE-EU). Disponível em: https://clinicaltrials.gov/ct2/show/NCT03461289. [Acesso em 19 jul. 2019].

17. NCT03421977. ClinicalTrials.gov. Long-Term Follow-up Study for Patients From AVXS101-CL-101 (START). Disponível em: https://clinicaltrials.gov/ct2/show/NCT03421977 [Acesso em 19 jul. 2019].

18. NCT03505099. ClinicalTrials.gov. Pre-Symptomatic Study of Intravenous AVXS-101 in Spinal Muscular Atrophy (SMA) for Patients With Multiple Copies of SMN2 (SPR1NT). Disponível em: https://clinicaltrials.gov/ct2/show/NCT03505099 [Acesso em 19 jul. 2019].

19. NCT03837184. ClinicalTrials.gov. Single-Dose Gene Replacement Therapy Using for Patients With Spinal Muscular Atrophy Type 1 With One or Two SMN2 Copies. Disponível em: https://clinicaltrials.gov/ct2/show/NCT03837184 [Acesso em 19 jul. 2019].

20. NCT03955679. ClinicalTrials.gov. AveXis Managed Access Program Cohort for Access to AVXS-101. Disponível em: https://clinicaltrials.gov/ct2/show/NCT03955679 [Acesso em 19 jul. 2019].

21. NCT03381729. ClinicalTrials.gov. Study of Intrathecal Administration of AVXS-101 for Spinal Muscular Atrophy (STRONG). Disponível em:

https://clinicaltrials.gov/ct2/show/NCT03381729 [Acesso em 19 jul. 2019].

22. Catalogue of Bias Collaboration. Catalogue Of Bias. [Internet]. Catalogue Of Bias. 2019. Disponível em: https://catalogofbias.org/biases/attrition-bias/ [Acesso em 27 jun. 2019].

23. Moher D, Pham B, Jones A, Cook DJ, Jadad AR, Moher M, et al. Does quality of reports of randomised trials affect estimates of intervention efficacy reported in meta-analyses? Lancet [Internet]. 1998 Aug 22 352(9128):609-13. Disponível em: http://www.ncbi.nlm.nih.gov/pubmed/9746022 [Acesso em 10 jul. 2019]. 
24. Schulz KF, Chalmers I, Hayes RJ, et al Empirical evidence of bias. Dimensions of methodological quality associated with estimates of treatment effects in controlled trials. JAMA J Am Med Assoc [Internet]. 1995 273(5):408-12. Disponível em: http://jama.amaassn.org/cgi/doi/10.1001/jama.273.5.408 [Acesso em 10 jul. 2019].

Como citar esse artigo:

Rieira R, Bagattini AM, Pachito D. Eficácia, segurança e aspectos regulatórios dos medicamentos órfãos para doenças raras: o caso Zolgensma ${ }^{\circledast}$. Cadernos Ibero-Americanos de Direito Sanitário. 2019 jul./set.; 8(3): 48-59.

http://dx.doi.org/10.17566/ciads.v8i3.538 